\title{
Novel Multi Parameter Sensor Approach for Interface Measurement
}

\author{
Gorenflo, Stefan and Schroth, Herbert \\ Endress+Hauser $\mathrm{GmbH}+\mathrm{Co}$. KG \\ Hauptstraße 1, 79689 Maulburg, Germany
}

\begin{abstract}
A new method to measure the interface of two separated liquids in a vessel is presented, where two independent measurement principles, namely the Time-Domain Reflectometry (TDR) and the capacitance measurement are combined in one device. This method needs only minimal effort for hardware, since the same probe can be used for both measurement principles. On the other hand it yields a wealth of complementary information for the signal evaluation. This information is used to make the measurement more robust: The determination of both, level and interface position under difficult conditions like presence of emulsion or foam and varying dielectric constants of the media is now possible, where one measurement principle alone would not deliver satisfying results. Additionally, in case of clearly separated media, the information is used for self calibration, so that knowledge of the dielectric constant is not necessary for device setup. Plausibility checks are possible to increase measurement reliability as well as self diagnostics to detect buildup of process media on the probe.
\end{abstract}

\section{Introduction}

The position of liquid media interfaces is an important process control parameter in chemical and petrochemichal industry, e.g. in oil-water separators. Methods based on density (measured with a float, differential pressure or gamma ray absorption), capacitance (measured with an isolated probe) or TimeDomain Reflectometry (TDR, measurement of the time of travel of high frequency pulses guided along a probe) are commonly used [1].

Among these the capacitance approach is the simplest way to measure the interface position. A coaxial, isolated probe is inserted into the vessel and the capacitance of the fluid entering the coax is measured. For media with high dielectric constant or conductive media, like water, a high capacitance change is observed, which is basically the capacitance of the probe isolation and thus is independent of media properties. For media with low dielectric constant, like hydrocarbons, only a small change in capacitance is observed, which is often neglected. The capacitance measurement has the advantage of beeing nonsensitive to emulsification, since only an integral value is measured that approximately corresponds to the capacitance of the same media with clear-cut interface. However, only one value can be measured, associated with the interface position, and the overall level remains undetermined.

In contrast, with the TDR principle, the overall level position and the interface position can be measured simultaneously. High frequency pulses are coupled on a probe and propagate along the probe as surface wave with nearly speed of light. At any change in dielectric constant along the probe, part of the signal is reflected and travels back to the receiver circuitry while part of the signal ist transmitted in the lower medium, where further reflections may occur. Thus, the measured reflection curve represents the change in dielectric constant along the probe, where the time between transmission and receiving of specific high frequency reflections (see Figure 1 using the example of an oil/water interface application). Knowing the dielectric constant $\varepsilon$ of the upper medium, the propagation time of the reflected pulses $\left(t_{\text {oil }}, t_{\text {water }}\right)$ can be converted to height information about the layers $\left(\mathrm{H}_{\text {oil }}, \mathrm{H}_{\text {water }}\right)$. Obviously, for determination of the interface position both echos from the TDR signal must be clearly detectable, otherwise one input parameter for calculation of the interface thickness would be missing. Unfortunately, the echo from the interface layer may vanish e.g. due to highly attenuating media or in case of heavy emulsion. Another drawback is that a varying dielectric constant of the upper medium will corrupt the measurement performance and accuracy, since it influences the propagation speed. 


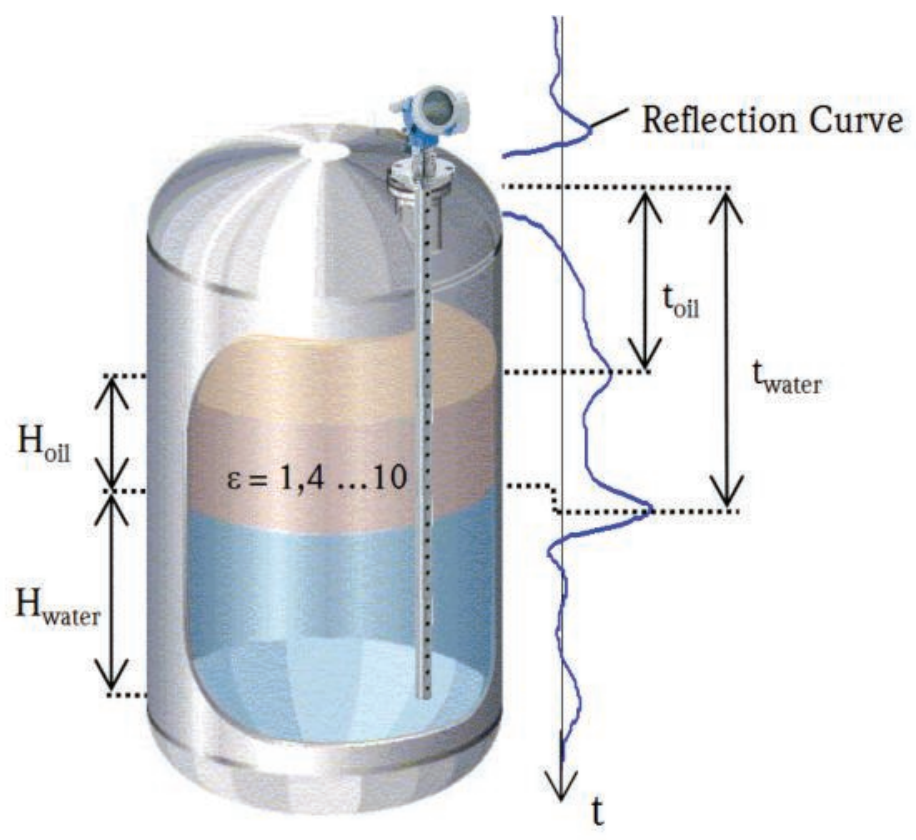

Figure 1: Schematic sketch of an interface application using a TDR system.

To solve the problems described above we suggest a multi parameter sensor approach combining all the advantages of the capacitance and the TDR measurement in one device [2]. Once a parameter in the TDR signal is missing, the information is delivered from the capacitance measurement. Even if clearly separated layers are available, the redundant capacitance information can be used to determine e.g. the dielectric constant of the upper liquid. Applications, where medium properties change during processing, can be solved without a new parametrization of the device. Furthermore, a variety of additional plausibility checks or diagnostics described in the following sections is possible.

\section{Technical Realization}

A block diagram of the multi parameter sensor circuitry is depicted in Figure 2. Standard TDR circuitry is used. The frequency of a $3.6 \mathrm{MHz}$ quartz transmit oscillator signal (TXO) is divided by a factor of 8 in a programmable logic device (CPLD) to get a pulse repetition frequency of $450 \mathrm{kHz}$ which triggers the pulse generator stage. High frequency pulses are generated there, with a pulse spectrum between $10 \mathrm{MHz}$ and $1.5 \mathrm{GHz}$, and are coupled on the probe by means of a directional coupler and a high pass filter [3]. Reflected pulses are received by a sampling circuit, consisting of a second, slightly misaligned quartz receive oscillator $(\mathrm{RXO})$ and pulse generator, which triggers a sampling diode. Sampled baseband signals then pass through an amplifier and anti-aliasing filter and an AD-converter delivers the digital reflection curve (echo curve) to a microcontroller, where the signals are evaluated together with the corresponding capacitance value using special signal processing algorithms.

Also common capacitance circuitry is used: An oscillator couples a $0.5 \mathrm{MHz}$ sinusoidal signal across a low pass filter on the probe [3]. The imaginary part of the probe impedance is calculated out of the measured voltage and current and gives the probe capacitance.

Both measurements use the same isolated coaxial probe, consisting of an inner metallic electrode, covered with a thin PFA layer and an outer tubular ground electrode, realized as perforated metallic tube directly attached to the sensor or as a bypass of the vessel. The probe measures the capacitance of the medium and also acts as wave guide for the electromagnetic pulses. 


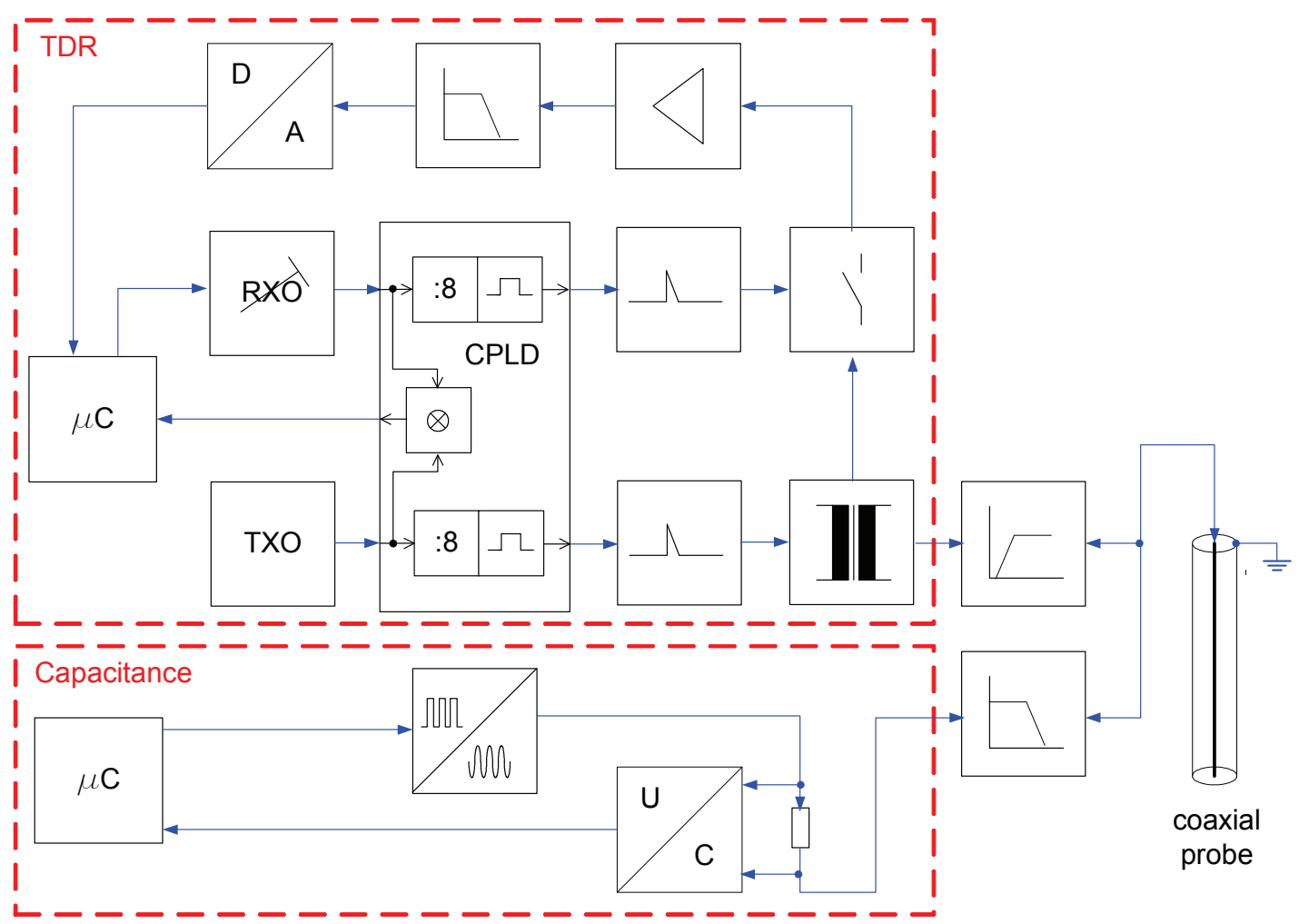

Figure 2: Block diagram of the sensor circuitry. Standard TDR and capacitance signals are coupled via filters on a coaxial probe [3].

Mathematically, the multi parameter sensor system has three free variables, the heights $H_{\text {oil }}, H_{\text {water }}$ and the dielectric constant $\varepsilon$ of the upper, nonconductive medium. Since also three equations can be formulated for the case where the TDR detects two echos, the system of linear equations is definite or even over-determined, if $\varepsilon$ is known and constant (the reference to oil and water is only to get convenient parameter designations, the equations are true for all media):

$$
\left\{\begin{array}{l}
H_{\text {oil }}=0.5 \cdot\left(t_{\text {water }}-t_{\text {oil }}\right) \cdot v_{\text {oil }} \\
H_{\text {water }}=D_{0}-0.5 \cdot t_{\text {oil }} \cdot v_{\text {air }}-0.5 \cdot\left(t_{\text {water }}-t_{\text {oil }}\right) \cdot v_{\text {oil }} \\
C_{0}+a \cdot H_{\text {water }}+b \cdot H_{\text {oil }}=C
\end{array}\right.
$$

where the following abbreviations are used:
a: $\quad$ Specific capacitance of water
b: $\quad$ Specific capacitance of oil
$\mathrm{C}_{0}$ : $\quad$ Capacitance of the uncovered probe
C: $\quad$ Measured capacitance
$\mathrm{D}_{0}$ : $\quad$ Probe length
$\mathrm{H}_{\mathrm{oil}}$ : Thickness of oil layer
$\mathrm{H}_{\text {water: }}$ : Thickness of water layer
$v_{\text {air: }}$ : Propagation velocity in the coaxial system filled with air
$\mathrm{v}_{\mathrm{oil}}: \quad$ Propagation velocity in the coaxial system filled with oil
$t_{\text {oil }}$ : Time of travel to the reflection from the oil layer
$t_{\text {water }}$ : Time of travel to the reflection from the water layer

In first approximation, the specific capacitance $a$ is only related to geometrical probe parameters, since effectively the capacitance of the probe isolation is measured, and the specific capacitance $b$ is approximately proportinal to the dielectric constant $\varepsilon$ of the upper medium. A more sophisticated model may be used to increase accuracy and universality, where a series connection approach is used for the 
isolation capacitance and the capacitance of the medium filled gap under consideration of medium conductivity and dielectric constant. The velocity $\mathrm{v}_{\mathrm{oil}}$ is inversely proportional to $\sqrt{\varepsilon}$, while $\mathrm{v}_{\text {air }}$ is taken constant.

Application of equation (1) is done in practice by case differentiation in the firmware algorithms, where the number of found TDR echoes, the measured capacitance value as well as historical measurement data is considered to select the appropriate case out of 18 relevant cases. This will be now explained for some common examples.

If, for example, one TDR echo is not detectable anymore (e.g. caused by foam on the surface, microwave absorption in the upper medium, or an emulsion layer between both media), the remaining echo is in a first step assigned either to the overall level or to the interface position by plausibility considerations taking into account the capacitance information and the echo position. In case the remaining echo belongs to the overall level, then the system of linear equations (1) changes to:

$$
\left[\begin{array}{ll}
a & b \\
1 & 1
\end{array}\right]\left(\begin{array}{c}
H_{\text {water }} \\
H_{\text {oil }}
\end{array}\right)=\left(\begin{array}{c}
C-C_{0} \\
D_{0}-t_{x}
\end{array}\right) .
$$

If one of the obtained layer thicknesses $\mathrm{H}_{\text {water }}$ and $\mathrm{H}_{\mathrm{oil}}$ becomes negative, the basic assumption about the origin of the reflected echo was wrong and the echo was actually reflected from the interface layer. On that condition, equation (1) yields:

$$
\left[\begin{array}{cc}
a & b \\
1 & 1-\frac{v_{\text {air }}}{v_{\text {oil }}}
\end{array}\right]\left(\begin{array}{c}
H_{\text {water }} \\
H_{\text {oil }}
\end{array}\right)=\left(\begin{array}{c}
C-C_{0} \\
D_{0}-t_{x}
\end{array}\right)
$$

In the case, where both liquids are clearly separated and two TDR echoes are detected, the capacitance value can be considered as redundant information, which is used to determine internal system parameters. In particular, the dielectric constant $\varepsilon$ of the upper medium is an interesting parameter, since it is directly involved in calculating the interface thickness depending on $v_{\text {oil }}$. The corresponding multi parameter system is then given as:

$$
a H_{\text {Water }}\left(H_{\text {oil }}(\varepsilon)\right)+b(\varepsilon) H_{\text {oil }}(\varepsilon)-C+C_{0}:=0 \text {. }
$$

This is a simple root finding problem, which can be solved with respect to $\varepsilon$ using numerical methods. Whenever the dielectric constant varies, e.g. due to process influences or altering media, the system automatically computes the correct distance to the interface layer without any information about $\varepsilon$ from the user.

In case $\varepsilon$ is known and the whole information of both measurements is available, the over-determined system in equation (1) can also be applied for self calibration. That means, the TDR and the capacitance measurement are always balanced. When the interface information of the TDR measurement is lost, the device switches over to the capacitance measurement without any discontinuities in the output signal. For this operating mode, an analogue equation to equation (4) can be readily formed.

In some applications it may be advantageous to entirely ignore the interface information from the TDR signal, since the echo constellation is ambiguous, e.g. due to multiple layers or interference echos caused by other installations in the vessel. In such an operating mode the overall level is determined by the first found TDR echo and the interface is always calculated using the capacitance value and therefore misleading echo interpretations are prevented.

We also use the capacitance for checks on plausibility of the TDR echos. For instance, a multiple echo of the upper medium, caused by back and forth reflection at the device flange and the medium, can clearly be distinguished from a situation with echoes from both media by the capacitance measurement. In a TDR system alone, such basic checks on reasonability are usually implemented using amplitude relations of the echos or plausibilities derived by the end-of-probe signal. Using the capacitance information, 
plausibility checks are performed with a completely independent measurement principle, which enormously increases reliability.

The multi parameter system can additionally be applied for diagnostic purposes, like detection of medium build-up along the probe or changes in the observed process. Here, the misbalance of the two independent measurement methods is intentionally used to generate a maintenance message to the operator, when the discrepancy of the TDR and capacitance measurement exceeds a certain threshold.

\section{Experimental Results}

Numerous tests were carried out, both, in laboratory and in real industrial environments with different media combinations. In particular, when emulsion layers arise, the advantages of the multi parameter sensor become clearly evident. A comparison of a multi parameter interface measurement with a standard TDR interface measurement in the laboratory is shown in Figure 3. Due to a highly attenuating upper medium, the interface echo is not detectable from time to time. The overall level (black curve) is reliably measured with both measurement approaches. The interface is erroneous for the TDR measurement alone (blue curve, slightly vertically shifted for better reading), whereas the multi parameter system delivers the correct distance to the interface layer using the capacitance information and the beforehand automatically determined dielectric constant (red curve).

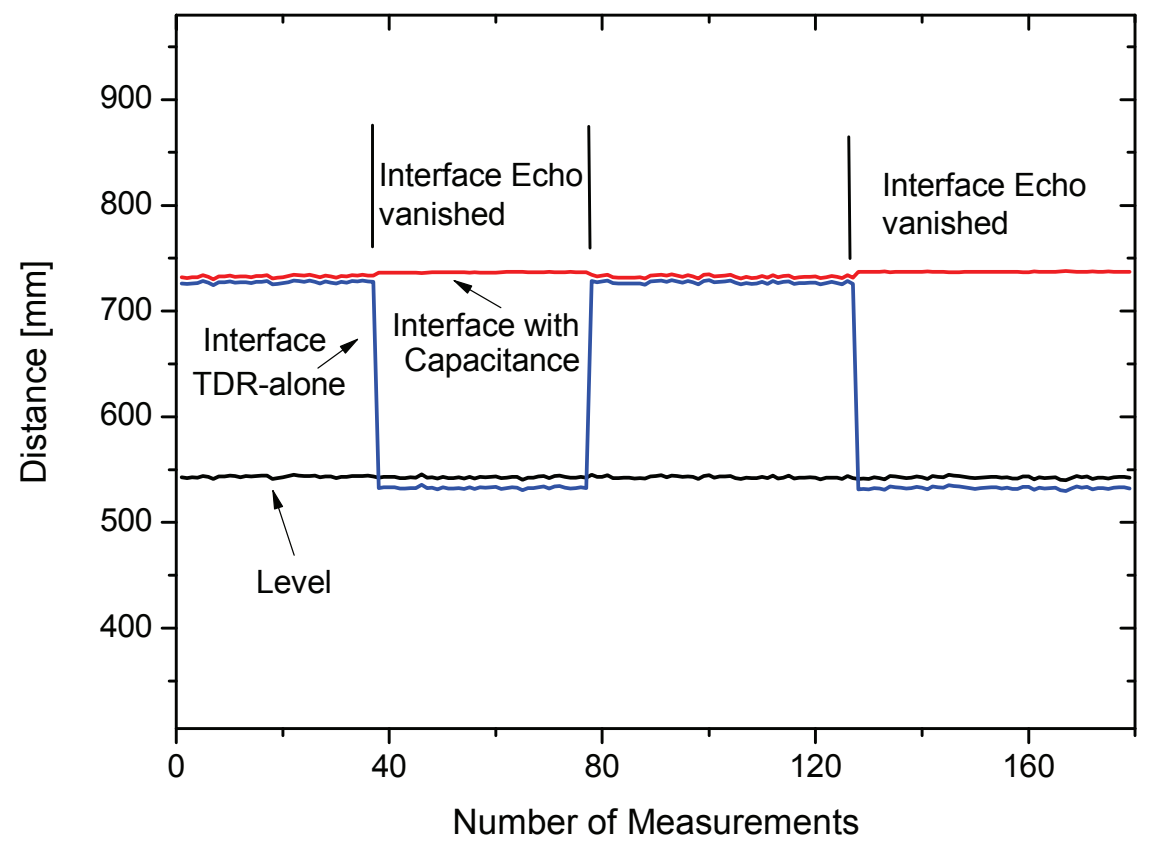

Figure 3: Comparison of a multi parameter interface measurement with a standard TDR interface measurement. In case the interface echo is lost, the TDR measurement delivers the same distance to the upper and lower medium, respectively. The multi parameter sensor still measures the correct level and interface.

The benefit of a self-calculating dielectric constant could be directly demonstrated in an oil/water separator in a refinery, where the composition of the hydrocarbon compound changed over time, leading to a varying dielectric constant between 5 and 15 . Up to now, such applications had to be solved using an independent measurement device to determine the dielectric constant of the medium for calibration of the interface measurement.

The multi parameter sensor has the potential to open up complete new fields of applications, which could not be satisfyingly measured with one measurement approach alone, or at least only with very expensive alternative methods (e.g. gamma ray absorption with several detectors along the vessel to overcome problems with emulsion layers). 


\section{Conclusion}

In summary, we developed a new interface measurement device based on TDR and capacitance technology. Beside the significantly enhanced measurement reliability and robustness on process impacts, due to the complementary measurement principle, a variety of additional useful functions for diagnostics and self-calibration were implemented. In numerous tests, the high potential of the multi parameter sensor for industrial interface applications could be evidently demonstrated. Especially with emulsion layers and with varying dielectric constants the multi parameter sensor proved to be advantageous as compared to a single TDR or the capacitance device.

\section{References}

[1] Wim van de Kamp: The theory and practice of level measurement, published by Endress+Hauser B.V., Postbus 5102, 1410 AC Naarden, Netherlands, 16. edition (2001)

[2] A. Malinovskiy, H. Schroth, D. Spanke: Method for filling level measurement, International patent application WO 2009077434

[3] R. Armbruster, R. Grozinger, B. Michalski: Device for determining and/or monitoring at least one fill level of at least one medium in a tank using a run-time measurement method and/or a capacitive measurement method, International patent application WO 2009077435 